\section{P-135 EVALUATION OF TOLERABILITY AND DEPRESCRIBING OF ANTI-FIBROTICS IN PULMONARY FIBROSIS (IPF) PATIENTS}

Sharon Chadwick. Hospice of St Francis, Berkhamsted, UK

\subsection{6/bmjspcare-2019-HUKNC.158}

Background IPF is a progressive fibrotic lung disease of unknown cause. Median survival for IPF patients is approximately three years from diagnosis. NICE guidance states that best supportive care should be offered from diagnosis to provide information, symptom relief and support withdrawal of therapies suspected to be ineffective or causing harm, and to provide end of life care (NICE, 2013). To this end, a hospice-based pulmonary fibrosis support group has been set up providing care for patients with IPF to improve access to palliative care support including consultant review. The antifibrotics nintedanib and pirfenidone (NICE, 2016; NICE, 2018) are recommended by NICE to slow the progression of the disease but have considerable side effects.

Aim This evaluation will collect data regarding the demographics of IPF patients seen, the source of referral and assess the proportion taking anti-fibrotics. The side effects experienced by patients will be described and obstacles in deprescribing anti-fibrotics outlined.

Methods A retrospective case note review will be performed to extract data from the narrative. This will be used to identify whether there are certain characteristics that these patients share in order to inform decisions regarding those who may be unable to tolerate anti-fibrotics in the future.

Result It is anticipated that many patients taking anti-fibrotics will experience significant side effects. There may be shared characteristics that enable us to predict which patients cannot tolerate these drugs that can have a significant and detrimental effect on quality of life of patients.

Conclusions The hospice has a highly evaluated IPF support group that provides professional and peer support to patients/ families. It facilitates access to the Palliative Medicine Consultant who is able to support patients in discontinuing antifibrotic drugs that can adversely affect quality of life. The characteristics of patients who do not tolerate anti-fibrotics are outlined and obstacles in deprescibing described.

\section{P-136 IMPLEMENTATION OF ELECTRONIC PRESCRIBING AND MEDICINES' ADMINISTRATION (EPMA) INTO A HOSPICE SETTING}

${ }^{1}$ Katherine Oakley, 'Lynda Simpson, ${ }^{1,2}$ Deborah Smart, 'Karen Causton, 1,2 Anne Regan, ${ }^{1}$ Sue O'Neill. 'St Helena, Colchester, UK; ${ }^{2}$ East Suffolk and North Essex NHS Foundation Trust, Colchester, UK

10.1136/bmispcare-2019-HUKNC.159

Background ePMA is used in many UK hospitals, but not in hospices. It can reduce prescribing errors, enable more efficient administration of medicines and free up staff to spend more time with patients (Ahmed, Garfield, Jani, Jheeta et al., 2016). St Helena introduced SystmOne into clinical practice in 2012 and implemented ePMA into the inpatient unit (IPU) in November 2018.

Aims To reduce drug errors, improve patient safety, strengthen information governance, and enable staff to use their time more efficiently.
Methods A project team of IPU and SystmOne managers, senior pharmacy staff and a consultant was set up and preimplementation research undertaken, including visits to other healthcare settings. A business plan highlighted training needs and cost implications. Specific formularies were built, mobile 'computers on wheels' (COWs) were ordered, the risk register was updated and training sessions for all clinical and pharmacy staff (group and 1-2-1) were delivered. We initially implemented ePMA incrementally as each new patient was admitted, and provided increased technical support to staff, including at evenings and weekends.

Results We identified that $40 \%$ of our recorded drug errors may not occur with ePMA, for example lost drug charts. We remained vigilant for new or unanticipated errors, and dealt with them swiftly.

Since implementation, the number of drug errors has fallen by $35 \%$ (from 28 to 18). A small increase level 2 errors (Hospice UK, 2017), from 3 to 4 has been noted, none related directly to ePMA.

Informal feedback from staff and patients has been positive, e.g. nurses have more ward time, patients do not feel the COWs detract from their interactions with staff.

Conclusion The system is now fully embedded in the inpatient setting. This year we plan to complete an audit, support other interested hospices, carry out staff and patient satisfaction surveys and implement discharge medication functionality.

\section{P-137 IMPLEMENTING E-PRESCRIBING - THE EXPERIENCE OF ST CATHERINE'S HOSPICE PRESTON}

Tracy Parkinson, Andrew Fletcher, Jimmy Brash. St Catherine's Hospice, Preston, UK

10.1136/bmjspcare-2019-HUKNC.160

Background E-prescribing is understood to provide benefits to patient safety by reducing medication errors and may provide some staff efficiencies (Ammenwerth, SschnellInderst, Machan, \& Siebert, 2008; Franklin, O’Grady, Parastou et al., 2007). However implementing e-prescribing, requires introduction of new working processes and within a specialist health care setting an adapted e-prescribing system is recommended (Ward \& Watson, 2013). Despite the perceived benefits, e-prescribing use nationally is low and a paucity in the research exists regarding implementation within a hospice setting.

Aims

- To share the lived experience of implementing e-prescribing within a hospice In-Patient Unit, Community team and Lymphoedema service;

- To monitor staff attitudes and perceptions of e-prescribing pre- and post- implementation;

- To review medication error rates pre - and post implementation.

Method Implementation commenced on 29 May 2019. The hospice has an established electronic patient record system and the e-prescribing software is an add-on to the existing system. The e-prescribing software has been specifically written for a hospice service. Implementation was supported by a contracted IT team and a designated project manager. A preimplementation questionnaire was completed by all hospice staff. The questionnaire focused on perceived benefits and barriers of e-prescribing, compared to the previous paper- 